\title{
Preliminary study on the correspondence of turning points between global flood occurrences and polar motion
}

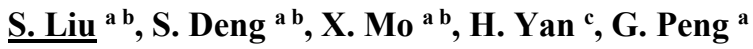 \\ ${ }^{a}$ Key Laboratory of Water Cycle and Related Land Surface Processes, Institute of Geographic Sciences and \\ Natural Resources Research (IGSNRR), Chinese Academy of Sciences (CAS), Beijing 100101, China; ${ }^{b}$ \\ University of Chinese Academy of Sciences (UCAS), Beijing, China; ${ }^{c}$ State Key Laboratory of Geodesy and \\ Earth's Dynamics, Institute of Geodesy and Geophysics, CAS, Wuhan , 430077 China \\ Email:liusx@igsnrr.ac.cn
}

\begin{abstract}
Floods, as one of the extreme hydrological events, develop at the Earth's surface much often than before, most of which have brought big losses to our society. Polar motion, as one of the Earth's motion factors, is largely excited by mass redistribution on earth, including flooding. The relationship between global flood occurrences and polar motion is explored from the correspondence of turning points. A moving average method with a subset size, which is equal to the prominent period, is suggested to acquire the turning point of polar motion. The combined method of moving t-test and accumulated anomaly is used to acquire the turning points of global flood occurrences. It is seen that the moving average method is more efficient to display the turning points in polhody than the method of removing periods as used elsewhere popularly. 2006 is a most obvious turning point both at polhody and the evolution of global flood occurrence. The variation in 1990s in polar motion also corresponds to that of global flood occurrence. These established relationships found between polar motion and flood occurrence in turning point encourages us to explore the role of excitation of floods to polar motion at least in the turning points in flood occurrences.
\end{abstract}

Keywords: $\quad$ Polar motion, flood occurrences, moving average, period 
Liu et al., Preliminary study on the correspondence of turning points between global flood occurrences and polar motion

\section{INTRODUCTION}

Hydrological extremes have brought human big disasters in the past (Hirabayashi et al., 2013) and the risk is projected to increase in the future (Muis et al., 2015). Recently Schumannn et al. (2016) gave a rethink to floods. Further to these knowledges, few has paid attention to another role floods may play to our globe, that is, the excitation to the earth's polar motion. Polar motion of the Earth is the motion of the Earth's rotational axis relative to its crust. Although the change of polar motion is extremely small, the mass of the earth is $10^{6}$ orders of magnitude large than the mass of the atmosphere. This makes the role of polar motion non-negligible (Oort, 1987) based on the principle that the total angular momentum of the earth system, which includes that of the solid earth, atmosphere, oceans, ices and others, does not change with time if the external toques is negligible.

Atmospheric angular momentum whose orientation involves motion (wind) and mass (pressure) terms and ocean parameters, such as ocean bottom pressure and currents are two of the most significant influences on polar motion (Barnes et al. 1983; Wahr 1983; Chao and Au 1991; Dickey et al. 1993; Ponte et al. 1998;Gross et al. 2003; Zhong et al., 2006). However, it has been seen that the sum of the atmospheric angular momentum and oceanic angular momentum does not entirely explain the observed variations of polar motion (Ponte et al. 1998; Nastula and Ponte 1999; Brzezi'nski et al. 2009). The remaining power might be provided by the hydrological angular momentum (HAM). There were rich results on hydrological excitation to polar motion based on GRACE data and other global data including model reanalysis data, other satellite data on all the hydrological components including soil moisture, precipitation, snow, glacier and groundwater (see review research by e.g. Winska et al., 2017). Our previous studies show some links between polar motion and runoff (Peng et al., 1980; Wang and Liu, 2013; Liu et al., 2014).

Given so many signals involved in polar motion, we want to see if there are any corresponding signals between floods and polar motion in turning points. By finding the correspondence of the abrupt change between the number of global flood occurrence and polar motion, a possible relationship between polar motion and flood occurrences is explored.

\section{DATA AND METHODS}

\subsection{Data}

Polar motion is measured with respect to a reference frame in which the solid Earth is fixed, from a conventionally defined reference axis, with axis $\mathrm{X}$ being positive along $0^{\circ}$ longitude, axis $\mathrm{Y}$ being positive along $90^{\circ} \mathrm{W}$ longitude, and the CIO (Conventional International Origin) being the pole's average location from 1900 to 1905 . The unit of polar motion is milli-arc-seconds (mas, corresponding to $\sim 3 \mathrm{~cm}$ at Earth's surface). Observed record of polar motion (X, Y) can be obtained from the International Earth Rotation and Reference Systems Service (IERS) combined Earth Orientation Parameters (EOP) solutions $08 \mathrm{C0} 4$ and C01 data (available at http://hpiers.obspm.fr/eoppc/index.). The $\mathrm{C} 01$ data are from 1846 onwards with 0.1 year time intervals before 1890 and 0.05 year time intervals after 1890 . The C04 daily time series is from 1962 to the present. Although C01has longer data record, it has a higher uncertainty before 1962 as shown in Fig. 1. We will thus only use C04 in this paper. Flood occurence data, showing the total number of reported flood hazards worldwide for each year,from 1962 to 2014, are obtained from EM-DAT dataset (http://www.emdat.be/database). To match the time scale with flood data, the daily series of C04 polar motion data is averaged into annual series. 
Liu et al., Preliminary study on the correspondence of turning points between global flood occurrences and polar motion

\subsection{Methods}

The turning points of polar motion can be identified from polhody, he Earth's pole moves on the Earth's surface along the spiral curve. Because polar motion is affected by many factors including periodic factors. Some techniques have to be chosen in order to see the turning points clearly. We thus first calculate the prominent periods, and then seek a suitable approach to remove the regular signals of periods in order to get turning points.

Fourier analysis is used to estimate the major periods of polar motion by using a Fast Fourier Transform (FFT). For polar motion component X or Y, it can be written as a discrete Fourier series (Chapra, 2012):

$$
\mathrm{g}_{j}=\frac{1}{n} \sum_{k=0}^{n-1}\left[F_{k} e^{i k \omega_{o} j}\right] \quad \text { for } j=0 \text { to } n-1
$$

where $n$ is the total number of the sample; $j$ is the discrete times at which polar motion samples are taken; $g_{j}$ is the value of polar motion taken at time $_{j} ; F_{k}$ is the $k^{\text {th }}$ Fourier coefficient; $i=\sqrt{-1} ; \omega_{0}=\frac{2 \pi}{n}, \frac{n}{k}$ is the $k^{\text {th }}$ period, $\frac{k}{n}$ is the $k^{\text {th }}$ ordinary frequency. Through the least squares fit method and with the consideration to the periodicity and symmetry of trigonometric functions, the Fourier coefficients are computed by FFT using software MATLAB:

$$
F_{k}=\sum_{j=0}^{n-1}\left[\mathrm{~g}_{j} e^{-i k \omega_{0} j}\right] \text { for } k=0 \text { to } n-1
$$

The power is computed by summing the squares of the Fourier coefficients as

$$
P_{k}=\left|F_{k}\right|^{2} \text { for } k=0 \text { to } n-1
$$

Those with the maximum values of power correspond to the prominent periods. For better identifying the prominent periods, the variance contribution is also calculated to obtain the percentage of the variance of each period in total variance. The variance contribution is computed as

$$
V C_{k}=\frac{P_{k}}{\sum P_{k}} \quad \text { for } k=0 \text { to } n-1
$$

If a series include a trend it may produce a period being equal to its data length. Detrending is thus used before applying FFT to seek periods.

There are two methods used to seek turning points. One is to remove the periods directly from the original data. Another is to do a moving average, which is a kind of a low-pass filter used in signal processing and commonly used to smooth out the short-term fluctuations and highlight the more long-term change trend of a time series data set. The $l$-years moving average time series can be written as (Wei, 2007):

$$
\hat{x}_{m}=\frac{1}{l} \sum_{p=1}^{l} x_{p+m-1} \quad \text { for } m=1 \text { to } n-l+1
$$

where $l$ is the length of the moving average which is better an odd number. If $l$ is an even, the moving average must be the mean between every two adjacent numbers of the new time series calculated by Equation (5). We will compare the efficiency between these two methods.

The same can be done to global flood occurrence data to acquire the turning points. However, as the evolution of global occurrence is much smoother than polar motion, the turning point is directly detected by the accumulated anomaly analysis, peak detecting and a moving t-test method. For n-length time series $\mathrm{G}$, given a fixed subset size h, we get two data groups: $G_{1}, G_{2} \ldots G_{\mathrm{h}}$ and $G_{\mathrm{h}+1}, G_{h+2} \ldots G_{2 \mathrm{~h}}$ (namely $G_{\mathrm{r} 1}$ and $G_{\mathrm{r} 2}$ ). The $\mathrm{t}$ statistic of the moving t-test method to test whether the means of two groups are different can be calculated as follows (William et al., 2007):

$$
\mathrm{t}=\frac{\overline{G r_{1}}-\overline{G r_{2}}}{\sqrt{\frac{s_{G r_{1}}^{2}+s_{G r_{2}}^{2}}{2} \sqrt{\frac{2}{h}}}}
$$


Liu et al., Preliminary study on the correspondence of turning points between global flood occurrences and polar motion

where $s^{2}$ is the square deviation, the upper line represents the mean. Then both subsets are modified by "shifting forward" every one step gradually; that is, at each step, excluding the first data point and including the first data point in next step. At the $i$ th step we have time series $\mathrm{G}_{i}, \mathrm{G}_{i+1, \ldots}, \mathrm{G}_{i+h-l}$ and time series $\mathrm{G}_{\mathrm{i}+\mathrm{h}}, \mathrm{G}_{\mathrm{i}+\mathrm{h}+1}, . . \mathrm{G}_{\mathrm{i}+\mathrm{h}+\mathrm{h}-}$ 1 to be compared. The degrees of freedom for this test are $2 h-2$. In this study, with the consideration of the prominent period of polar motion, the value of $h$ is set to be 5.By comparing the turning points between polar motion and global flood occurrence, the relationship between the two is explored.

\section{RESULTS}

\subsection{The periodic analysis of polar motion}

The Fourier analysis result for the de-trending yearly data of polar motion component $\mathrm{X}$ is shown in Fig. $2 \mathrm{a}$.The first prominent period of polar motion is 6.625 years with the contribution to the total variance being $55 \%$ (Fig.2b). Fig.2c and Fig.2d shows the result for the original data of component X. It is seen that the first prominent period calculated is 52 years, which is equal to the data length. As this result contends the noise of trend, it is not a true period. The results of average yearly $\mathrm{Y}$ series with or without linear trend removed are almost the same as $\mathrm{X}$ series, which thus are not shown in the figure.
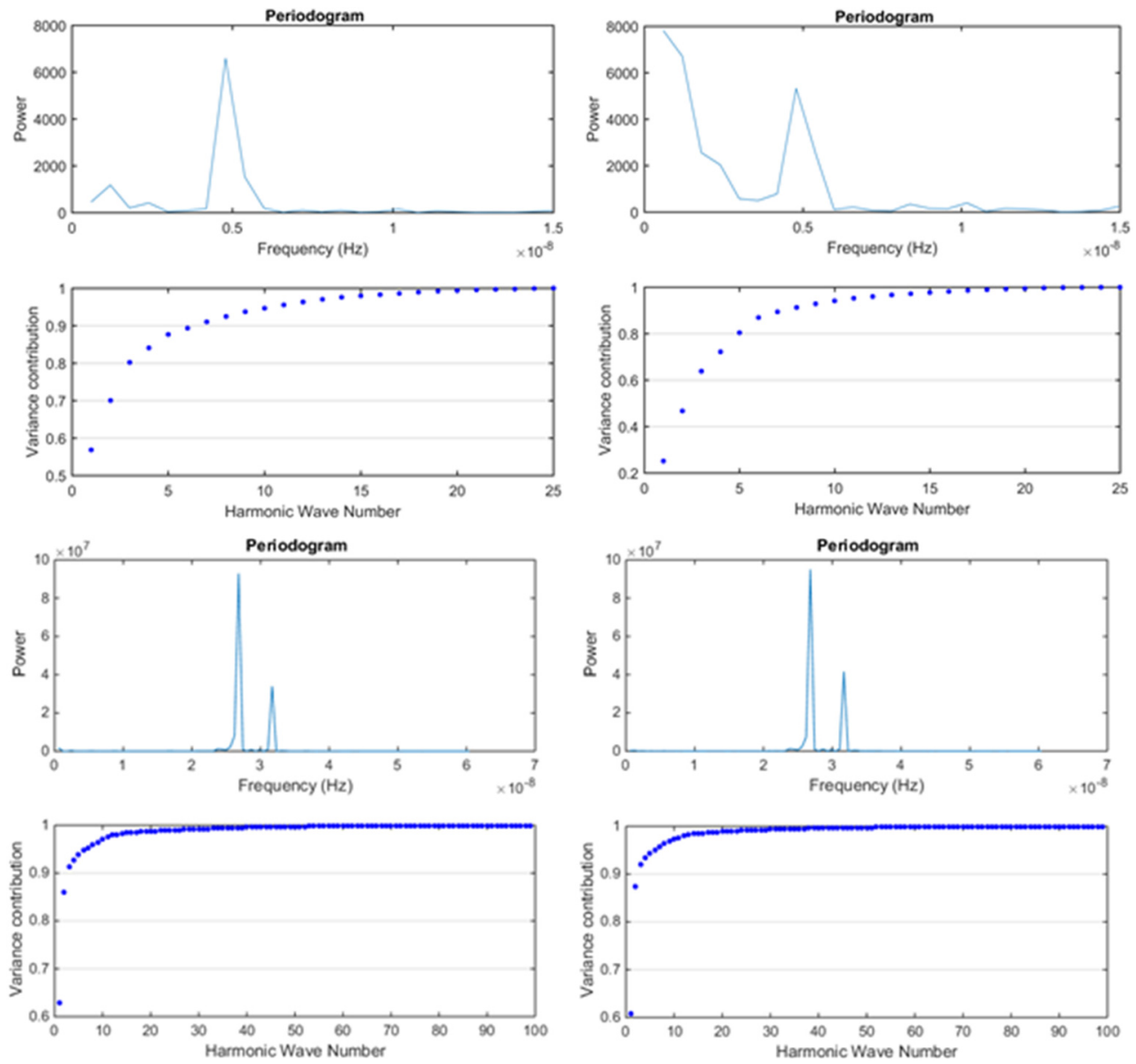

Figure 2. The results of Fourier periodic analysis: a) The power spectrum of yearly X series with linear trend removed ; b) The same as a) but for original yearly X series; c) The accumulative variance contribution of the first major periods of yearly X series with linear trend removed; d) The same as c) but for original yearly X series; e) The same as a) but for daily X series; (f) The same as a) but for daily Y series ; g) The same as c) but for daily X series; h) The same as c) but for daily Y series.

We also do the same for the daily data of polar motion (Fig. 2e $\sim \mathrm{h}$ ). The two prominent periods, corresponding to the first two maximum power values, are 1.18 and 1.00 years for component $\mathrm{X}$ and $\mathrm{Y}$ respectively. The accumulative variance contribution of the first two major periods is more than $80 \%$ for both components. The first prominent period, which is 1.18 year, encompassed over $60 \%$ of the total variation for both components. Period 1.18 corresponds to Chandler wobble (hereafter called CW) with a period of about 431.7 days, and period 1.00 corresponds to annual wobble (hereafter called AW). The prominent period of 6.625 for yearly 
Liu et al., Preliminary study on the correspondence of turning points between global flood occurrences and polar motion

a)

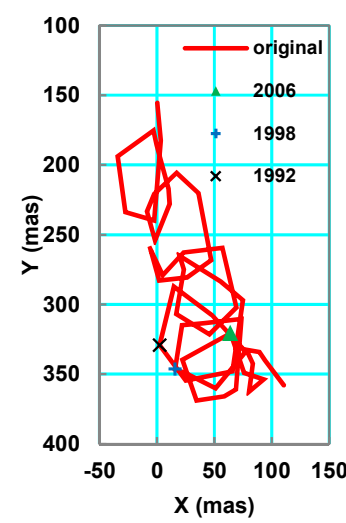

e)

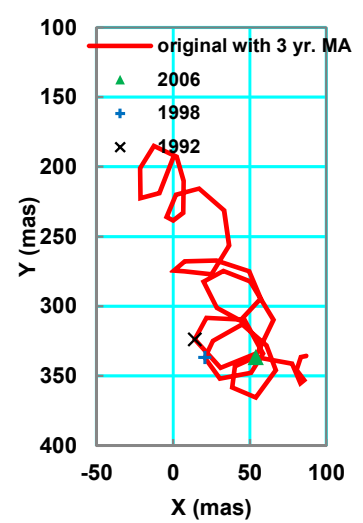

b)

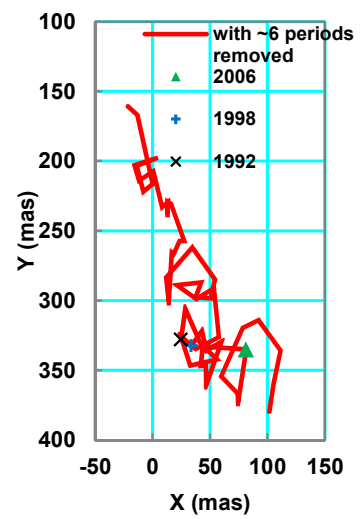

f)

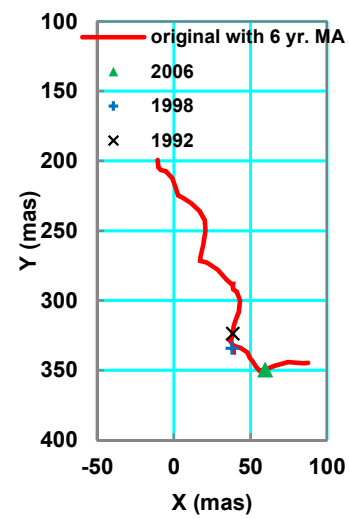

c)

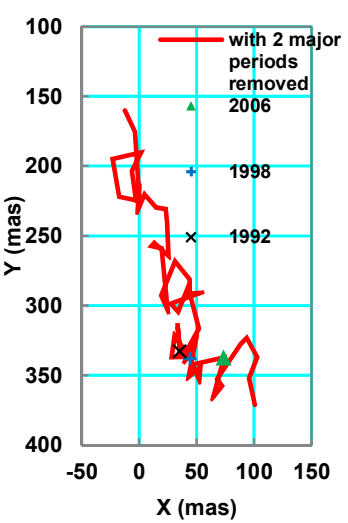

g)

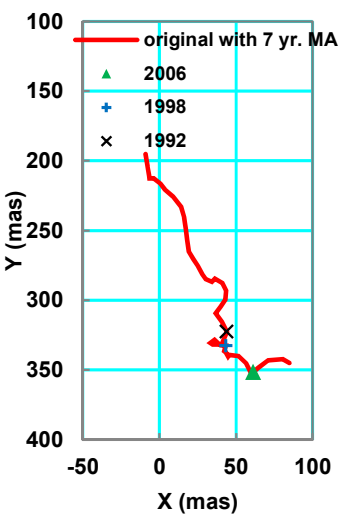

d)

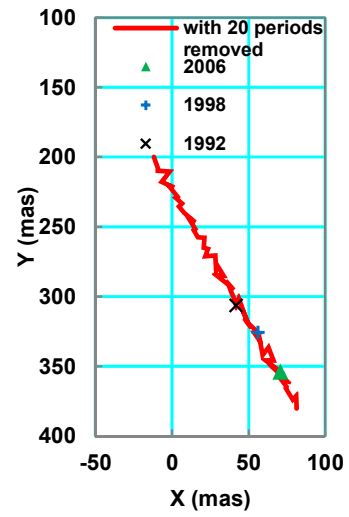

h)

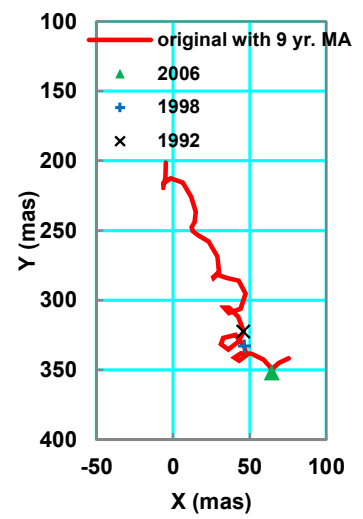

Figure 3. a) The track A which is based yearly averaged data of original C04 daily data; b) The track B which is track A with the prominent period of $\sim 6$ years removed; c) Track $C$ which is track A with 2 prominent periods removed; d) Track D which is track A with 20 periods removed; $(\mathrm{e} \sim \mathrm{h})$ The polyhodies which are track A with a moving average being done with the subset size being from 3, 6, 7 and 9 years respectively.

polar motion is the least common multiple of CW (1.2 years) and AW (1 year) and thus can be considered as the resonance of $\mathrm{CW}$ and $\mathrm{AW}$. The period of $\sim 6$ years was also found in other features of polar motion, such as the amplitude (Peng et al. 1980; Han et al., 2006) and velocity (Qian, 1995).

\subsection{The turning point of polar motion displayed by the polhody}

Polhody A as shown in Fig. 3a is based on original yearly data. We can see that there are so many signals combined together that it is hard to see any turning points from it. We then obtained polhody B (Fig. 3b) from polhody A by removing the period of 6.625 years identified in the above section. By comparing polhody A and polhody B, it sees polhody B displays a more prominent secular change but still has the disturbance of periods. This can be explained that besides the prominent period of 6.625 years, there are many other periods inside the polar motion. So we obtain polhody $\mathrm{C}$ and polhody D by removing 2 and more periods from polhody A, as shown in Fig. 3c and 3d. It is seen that with more periods removed, more approaching to its linear trend of the secular change of polar motion. However with more periods removed, more possible turning points are also removed together with the signal of periods. With all the trying by removing 1 to 20 periods, there is no way of showing the turning point (not fully shown in the figure) nicely.

We then try another way to seek turning point by doing a moving average to the yearly polar motion data with different average lengths. It is interesting to see from Fig.3e h that when the size of the subset increases by approaching to prominent period of 6 years, the turning point becomes obvious, away from the disturbance of any periods, which seems removing every 'circle' out and showing the turning points obviously. With the size of subset increasing again, some period cycles return again with more and more hiding signal of turning points. 
Liu et al., Preliminary study on the correspondence of turning points between global flood occurrences and polar motion

The polhody with a moving average at a prominent period of 6 years (Fig. 3f) is so far the best to show the turning points. As shown in Fig.3f, there is an obvious turning point in 2006 and a slightly less obvious turning point around 1990s. These turning points well correspond to previous finding by Lambert et al. (2006), Roy and Peltier (2011) and Chen et al. (2013).

\subsection{The turning points of floods}

From the record of global occurrences, it shows that the annual total number of reported floods worldwide (bar in Fig. 4) had gradually increased since around the middle of last century, reached the maximum in 2006, and

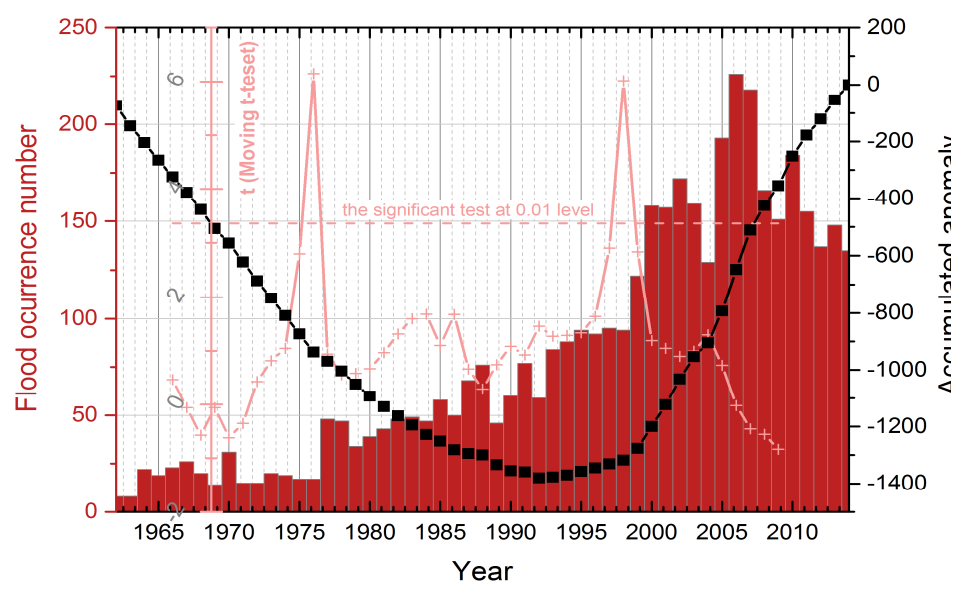

Figure 4. The statistical analysis results of global flood occurrence as shown in the bar. The light red curve with cross symbols is the result of $t$ time series calculated by Equation (6). If $t$ at a year passes the significant test at 0.01 which is the light red dash line, this year is a turning point. The black curve with square symbols is the curve of accumulated anomaly.

gradually decreased afterwards. Moving t-test results (line with cross in Fig. 4) show that 1976 and 1998 are turning points with abrupt changes. Accumulated anomaly (line with solid squares in Fig. 4) shows that the number of flood occurrences experienced a significant fluctuation in these 53 years. It gradually decreased since 1962 and reached minimum in 1992, then gradually increased, which means that the anomalies are negative before 1992 and are positive after 1992. Peak analysis, moving t-test and accumulated anomaly jointly indicate that there are turning point in global flood occurrence in 1990s and 2006.

\subsection{The correspondence of turning point between polar motion and flood occurrences and discussions}

From the above, we can see that the turning points of 2006 and 1990s in polhody are in agreement with the turning points of global flood occurrences. There are two possible explanations. On the one hand, the variability in terrestrial water storage plays an important role in inducing the secular changes of polar motion (Adhikari and Ivins, 2016), which means worldwide floods are also possible to induce sharp changes in polar motion. On the other hand, the increased ice melting rates and global warming could cause not only the turning point in polar motion (Cheng et al., 2011; Petit and Luzum, 2010; Chen et al., 2013; Roy and Peltier, 2011, Huang, et al., 2016) but also the flood hazard (Schiermeier, 2011).

We only use the number of flood events to explore the correspondence between polar motion and floods. The result has potential limitation as the data of flood occurrences used from the dataset may not able to entirely be related to changes of water volumes from the atmosphere to the land surface, as addressed by e.g Van Dijk et al. (2009). Further research will be undertaken to elucidate the potential links between polar motion and flood volumes as opposed to numbers.

\section{CONCLUSIONS}

We identify that period of $\sim 6$ years plays the dominant role of period in the secular change of polar motion. Taking a moving average with a suitable subset size equal to the prominent period of $\sim 6$ years can nicely show the turning point from the polhody. Removing periods directly from original data is too strong to show the turning points. It is found that the turning points in 1990s and 2006 in polar motion are in agreement with the turning points in reported flood occurrence worldwide. This link encourages us to do further work on the relationship between earth rotation and floods.

\section{ACKNOWLEDGMENTS}

This paper is financially supported by the 973 project (2012CB957802), project of Chinese National Natural Science Foundation $(41471026,31171451)$ and " 135 " Key Project for the Strategic Science Plan in IGSNRR, CAS. 
Liu et al., Preliminary study on the correspondence of turning points between global flood occurrences and polar motion

\section{REFERENCES}

Adhikari S, E. R. Ivins (2016). Climate-driven polar motion: 2003-2015. Science Advances, 2: 1-10

Barnes R. T. H. , R. Hide, A. A. White, C. A. Wilson CA (1983). Atmospheric angular momentum fluctuations, length-ofday changes and polar motion. Proceedings of the Royal Society of London, 387(1792): 31-73. doi:10.1098/rspa.1983.0050

Chao F. B., A. Y. Au (1991). Atmospheric excitation of the earth's annual wobble: 1980-1988. Journal of Geophysical Research: Solid Earth, 96(B4):6577-6582. doi:10.1029/91JB00041

Chapra, S. C. (2012). Applied numerical methods with MATLAB for engineers and scientists, pp.394. McGraw-Hill, New York.

Chen, J. L., C. R. Wilson, J. C. Ries, and B. D. Tapley (2013). Rapid ice melting drives Earth' s pole to the east. Geophysical Research Letters, 40, 1-6, doi:10.1002/grl.50552.

Cheng, M. K., J. C. Ries, and B. D. Tapley (2011). Variations of the Earth's figure axis from satellite laser ranging and GRACE. Journal of Geophysical Research, 116, B01409, doi:10.1029/2010JB000850

Dickey J.O., S. L. Marcus, C. M. Johns, R. Hide, S. R. Thompson (1993). The oceanic contribution to the earth's seasonal angular momentum budget. Geophysical Research Letters, 20(24):2953-2956. doi:10.1029/93GL03186

Gross R. S., I. Fukumori, D. Menemenlis (2003). Atmospheric and oceanic excitation of the earth's wobbles during 19802000. Journal of Geophysical Research: Solid Earth, 108(B8): 2370. doi:10.1029/2002JB002143

Gross, R. S., and M. Poutanen (2009). Geodectic observations of glacial isostatic adjustment. Eos Trans. AGU, 90(41), doi:10.1029/2009EO410004.

Han, Y., L. Ma, and Z. Yin (2006). Time-variation of periodic components of polar motion amplitude. Progress in Geophysics, 21(3): 798-801(in Chinese)

Hirabayashi, Y., R. Mahendran, S. Koirala, L. Konoshima, D. Yamazaki, S. Watanabe, H. Kim, and S. Kanae (2013). Global flood risk under climate change. Nature Climate Change, 3(9):816-821, doi:10.1038/NCLIMATE1911.

Hopfner, J. (2004). Low-frequency variations, chandler and annual wobbles of polar motion as observed over one century. Surveys in Geophysics, 25: 1-54

Huang, M., L. Zhu, H. Gong, Y. Shao (2016). Close correlation between global air temperature change and polar motion during 1962-2013. Journal of Geophysical Research-Atmospheres, 121(19).

Lambert, S. B., Bizouard C., Dehant V. (2006). Rapid variations in polar motion during the 2005-2006 winter season. Geophysical Research Letters, 33(13):5-6.

Liu S., S. Wang, Y. Wang, M. Li, and M. Huang (2014). Exploring the Relationship between Polar Motion and Runoff. Advances in Meteorological Science and Technology, 4(3):6-12 (in Chinese).

Oort, A. H. (1989). Angular momentum cycle in the atmosphere-ocean-soild Earth system. Bulletin American Meteorological Society, 70(10): 1231-1242.

Muis S., B. Guneralp, B. Jongman, J. C. J. H. Aerts, P. J. Ward (2015). Flood risk and adaptation strategies under climate change and urban expansion: A probabilistic analysis using global data. Science of the Total Environment, 538: 445457.

Nastula J., R. M. Ponte (1999). Further evidence for oceanic excitation of polar motion. Geophysical Journal International, 139(1):123-130. doi:10.1046/j.1365-246X.1999.00930.x

Peng G., W. Lu and Y. Yin (1980). Same problems about the earth pole shift and climate. Chinese Journal of Atmospheric Sciences, 4(4):369-378, doi:10.3878/j.issn.1006-9895.1980.04.11. (in Chinese)

Petit, G., and B. Luzum (2010). IERS Conventions (2010), pp.179. IERS Technical Note, 36.

Ponte R. M., D. Stammer, J. Marshall (1998). Oceanic signals in observed motions of the earth's pole of rotation. Nature, 391:476-479.doi:10.1038/35126

William H.; Saul A. Teukolsky; William T. Vetterling; Brian P. Flannery (1992). Numerical Recipes in C: The Art of Scientific Computing, pp.727. Cambridge University Press, Cambridge.

Qian C. (1995). The period analysis of polar motion speed. Annals of Shanghai Obeservatory, Academia Sinnia, 16: 3540. (in Chinese).

Roy, K., and W. R. Peltier (2011). GRACE era secular trends in Earth rotation parameters: a global scale impact of the global warming process? Geophysical Research. Letters, 38, L10306, doi:10.1029/2011 GL047282.

Schiermeier, Q. (2011). Increased flood risk linked to global warming. Nature, 470: 315-315.

Schumann, G. J. P., D. Stampoulis, A. M. Smith, C. C. Sampson, K. M. Andreadis, J. C. Neal, Bates, P. D. (2016). Rethinking flood hazard at the global scale. Geophysical Research Letters, 43: 10249-10256.

Wahr, J. M. (1983). The effects of the atmosphere and oceans on the earth's wobble and on the seasonal variations in the length of day - II. results. Geophysical Journal of the Royal Astronomical Society, 74(2):451-487. doi:10.1111/j.1365246X.1983.tb01885.x

Wang, S. and S. Liu (2013). Exploring the relationship between polar motion and a primitive river' $\mathrm{s}$ runoff based on Granger causality, IAHS Publ. 360:131-140.

Wei, F. Y. (2007). Statistical diagnosis and prediction technology of modern climate, pp.47. China Meteorological, Beijing. (in Chinese)

Winska, M., J. Nastula, D. Salstein (2017). Hydrological excitation of polar motion by different variables from the GLDAS models. Journal of Geodesy, 40(1792):1-13.

Zhong, M., H. Yan, X. Wu, J. Duan, and Y. Zhu (2006). Non-tidal oceanic contribution to polar wobble estimated from two oceanic assimilation data sets. Journal of Geodynamics, 41:147-154. 